\title{
Dynamic Multi-user MIMO Scheduling with Limited Feedback in LTE-Advanced
}

\author{
Meng Wang, Feng Li, Jamie S. Evans and Subhrakanti Dey \\ Department of Electrical and Electronic Engineering, University of Melbourne, Australia \\ Email: \{meng.wang, fenli, jse, sdey\}@unimelb.edu.au
}

\begin{abstract}
Multi-User MIMO (MU-MIMO) systems have gained numerous attention from researchers in the past decade due to its substantial gains in the system throughput. Most of the initial research assumes the knowledge of perfect channel state information at the transmitter (CSIT). However, this is considered impractical and research over the past few years has been focused on receivers feeding back limited information to the transmitter. In this work, we consider a transparent MU-MIMO system model with limited feedback. We propose a general framework for dynamic MU-MIMO scheduling with the capability to switch between Single-User MIMO mode and (Multi-Rank) Multi-User MIMO mode without users feeding back additional multi-user information. Specifically, this is done by each user carefully estimating its CQI under the hypothesis of Multi-Rank MultiUser MIMO transmission and taking advantage of the codebook structure. We consider the sum rate of the system assuming each user uses ML and LMMSE receiver and show that our proposed scheduler, with significantly reduced feedback load, outperforms the best-companion user pairing.
\end{abstract}

Index Terms-Multiuser-MIMO, Limited Feedback, Dynamic Scheduling, LTE

\section{INTRODUCTION}

In the last decade, the Multiple-Input Multiple-Output (MIMO) system has been studied extensively. The knowledge of channel state information (CSI) at the transmitter is critical for Multi-User MIMO, while it is not essential for Single-User MIMO [1]. CSI feedback allows the base station to adaptively transmit in the downlink based on users' instantaneous channel states. However, in practice, perfect CSI at the transmitter is impossible to obtain. In 3GPP Long Term Evolution (LTE), each user measures its channel through downlink reference signals and feeds back the CSI to the base station [2]. This includes feeding back three quantities to the base station scheduler, namely the rank index (RI), indicating the number of streams recommended by the user; the preferred precoding matrix index (PMI) from a set of pre-designed precoding codebooks, corresponding to the above RI; and a channel quality information (CQI) indicating the channel quality corresponding to the above RI and PMI. Currently, 3GPP is investigating how to enhance the performance of MU-MIMO and whether it is necessary to feedback multi-user information to enable MU-MIMO scheduling in LTE-Advanced.

When the number of users exceeds the number of antennas at the base station (BS), it is necessary for the BS to select a subset of users to communicate in a time/frequency resource, given the feedback from all the users. Rank-1 scheduling refers to every user supporting only a single data stream. To schedule rank-1 users, orthogonality between each user's channels is the key. With the assumption of perfect CSIT, the authors in [3] have proposed a Semi-orthogonal User Selection (SUS) algorithm. It is assumed in the paper that the CSI is known perfectly at the transmitter and also the number of receiving antenna is one for every user. When extended to users with multiple antennas, this algorithm simply treats each antenna as a separate user and performs antenna selection.

User scheduling with the assumption of limited feedback is considered in [4]-[8]. The most recent works in the literature assume that every receiver has the same number of receiving antennas (either 1 for MISO or $N$ for MIMO), and each user only supports 1 data stream (i.e., rank-1 transmission is assumed). For instance, the authors in [6] have proposed a user pairing scheme where the user pairing principle is to find the two users whose preferred precoding matrix are orthogonal. They assumed that each user has fixed number of receiving antennas and they are all of rank- 1 . The authors in [7] have proposed a joint user pairing scheme where the base station calculates estimated SNR for all users. This can be computationally expensive. They also assumed that each user has fixed number of receiving antennas of $N$ and they are all of rank-1. However, these assumptions are not true in practice as each user would have different number of receiving antennas and can support different transmission ranks.

The best-companion user pairing scheme was proposed in [9] to coordinate a pair of users from a single cell or multicell, where each user feedback an additional best-companion PMI. The goal here is to maximize the SINR or to minimize the interference between the two users. However, there are several problems with the best-companion user pairing. First of all, feedback overhead is high. Secondly, when there is small number of users, the BS scheduler may fail to find a best-companion pair. Finally, the best-companion user pairing can only schedule users of the same rank. The authors in [8] compared different PMI feedback schemes for MU-MIMO pairing. In particular, they introduced the idea of grouping the precoding matrices into clusters but they did not give any methods. We will propose a method of grouping the 4-Tx LTE codebook [2] into clusters which will be used later for CQI estimation.

In this paper, we will focus on multi-user dynamic scheduling and the necessity of feeding back multi-user information to support it. Our objective is to devise a dynamic multi-user scheduling scheme based on user RI/PMI/CQI without addi- 
tional MU-MIMO related feedback to support MU-MIMO. We assume that different users have different number of antennas and each may operate with different rank (less than or equal to the number of antennas). The main contributions of this paper are listed as follows;

- A clustering algorithm is proposed for the 4-Tx LTE codebook, which can be used for other codebooks as well. It is used for the purpose of CQI estimation to support MU-MIMO transmission.

- A dynamic MU-MIMO scheduler is proposed to enable dynamic switching between SU-MIMO and MU-MIMO.

- We focus on the achievable rate of the system and show that our proposed scheduler outperforms the bestcompanion user pairing with significantly reduced feedback overhead.

This paper is organized as follows. The system model is outlined in Section II. In Section III, we propose a clustering algorithm for the 4-bit 4-Tx LTE codebook and a CQI estimation method. In Section IV, we propose a dynamic MUMIMO scheduler with the capability to switch between SUMIMO and MU-MIMO based on users' feedback. Sum rate expression is derived in Section $\mathrm{V}$ and simulation results are presented in Section VI. Finally, we conclude with a brief summary of the key results in Section VII.

\section{System MODEL}

We consider a general MU-MIMO system shown in Figure 1 where the base station (BS) is equipped with $M$ transmit antennas and there is a total of $K$ users, where user $k \in$ $\{1,2, \ldots, K\}$ is equipped with $N_{k} \in\{1,2, \ldots, M\}$ receiving antennas. Let $\mathbf{H}_{k}$ denote the $N_{k} \times M$ MIMO channel between user $k$ and the base station, where we assume that the elements in $\mathbf{H}_{k}$ are independently and identically distributed (i.i.d.) circularly symmetric Gaussian random variables with zero mean and unit variance.

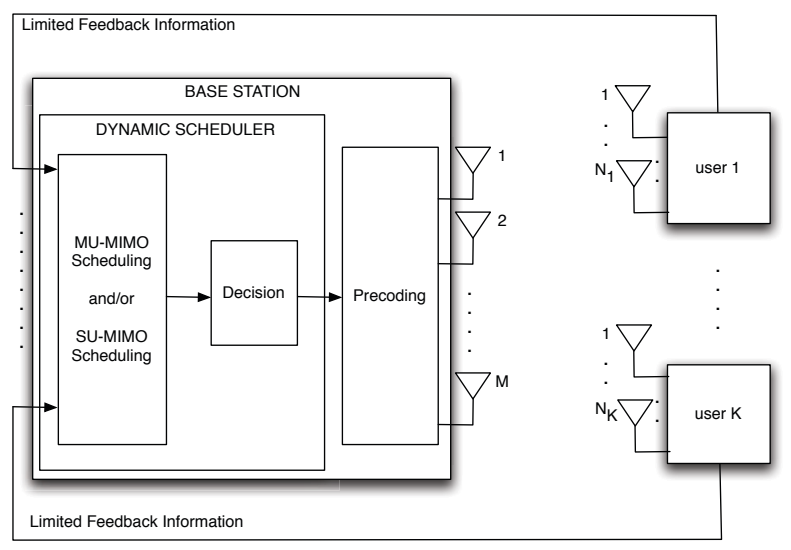

Fig. 1. System Model

We assume that the above MU-MIMO system can support multi-rank multi-user MIMO. (Notice that the multi-rank multi-user MIMO operation is made feasible in LTE Rel'9 via transmission mode 8 and is further enhanced in LTEAdvanced.) We further assume that each user can estimate the channel perfectly with the help of cell-specific reference signal (CRS) or user-specific demodulation reference signal (DMRS) and that a user is unaware of whether or not it will be co-scheduled with other users in the same time-frequency resource. The limited feedback scheme specified in the LTE standard [2] is adopted with only slight modification on CQI reporting scheme. The following paragraphs briefly describe how various feedback quantities are calculated by the user.

Each user $k$ first determines a preferred rank $R_{k} \in$ $\left\{1,2, \ldots, \min \left\{M, N_{k}\right\}\right\}$ to operate on. This can be done by first computing the singular value decomposition (SVD) of $\mathbf{H}_{k}^{H} \mathbf{H}_{k}$ and then selecting the number of dominant eigenvalue(s). For simplicity, we have set a threshold on the singular values and the rank index is the number of singular values above the threshold. For $M=4$, a user will require at most 2 bits for feeding back the rank index.

After user $k$ determines the rank index $R_{k}$, it will then determine a preferred PMI by computing

$$
\alpha(k)=\arg \max _{\mathbf{C} \in \mathcal{C}^{\left(R_{k}\right)}} \operatorname{det}\left(\mathbf{I}+\mathbf{H}_{k} \mathbf{C} \mathbf{C}^{H} \mathbf{H}_{k}^{H}\right),
$$

where $\mathcal{C}^{\left(R_{k}\right)}$ is the codebook of rank $R_{k}$. No matter how the base station constructs the precoding matrix (either to use a codebook based approach or to use a non-codebook based approach), users need to inform the base station of the channel direction information in the form of a preferred precoding matrix, which must be selected from a codebook. Intuitively, each user selects a precoding matrix from the codebook such that the rate is maximized. We use the 4-bit 4-Tx LTE codebook specified in [2] and each user will require 4 bits to feedback the preferred PMI.

Finally, each user has to feedback a channel quality indicator (CQI) to the base station. In LTE, the CQI indicates the recommended modulation scheme and coding rate [2]. In this paper, the performance measure is the sum rate, not the bit error rate, therefore a different quantity is used for the CQI. For SU-MIMO, CQI is usually chosen to be channel norm or SNR. However, for MU-MIMO system, feeding back the channel norm or SNR is not always desirable because of the possible interferences caused by other co-scheduled users. As a result, each user must estimate the interferences plus noise and feedback the estimated rate as a CQI measure. We will propose a CQI estimation method in the next section.

The base station performs scheduling and precoding based on the above feedback information from users (we assume that the precoding matrix is selected from the codebook $\mathcal{C}$ ). Let $\mathcal{U}$ represent the set of scheduled users on the same time/frequency resource. The $M \times R_{k}$ precoding matrix for user $k$ is denoted by $\mathbf{W}_{k}$. At user $k \in \mathcal{U}$, the received signal vector $\mathbf{y}_{k}$ can be written as

$$
\mathbf{y}_{k}=\mathbf{H}_{k} \rho \mathbf{W}_{k} \mathbf{x}_{k}+\sum_{\substack{j \in \mathcal{U} \\ j \neq k}} \mathbf{H}_{k} \rho \mathbf{W}_{j} \mathbf{x}_{j}+\mathbf{n}_{k},
$$


where the $R_{k} \times 1$ vector $\mathbf{x}_{k}$ represent the signal intended for user $k$. We assume that $\mathbf{E}\left[\mathbf{x}_{i} \mathbf{x}_{i}^{H}\right]=\mathbf{I}$ for $i \in \mathcal{U} . \mathbf{n}_{k} \sim$ $\mathcal{C N}\left(\mathbf{0}, \sigma_{n}^{2} \mathbf{I}\right)$ is the complex Gaussian noise. The parameter $\rho$ scales the transmit precoding matrices to satisfy the total transmit power constraint as follows,

$$
\rho^{2} \operatorname{Tr}\left(\sum_{i \in \mathcal{U}} \mathbf{W}_{i} \mathbf{W}_{i}^{H}\right)=P_{t} .
$$

\section{Codebook Clustering AND CQI Estimation}

\section{A. Codebook Clustering}

The purpose of codebook clustering is for reducing the complexity in CQI estimation; intuitively, users whose PMIs are close to each other will not be scheduled together. To measure the closeness of the precoding matrices, we will need to define a distance measure. For the 4-bit 4-Tx LTE codebooks, the columns of each precoding matrix forms an orthogonal basis and it spans a complex subspace. First we will define the principal (or canonical) angles between two complex subspaces spanned by two precoding matrices as follows.

Definition 1: Let $\mathcal{M}_{1}, \mathcal{M}_{2}$ be subspaces in $\mathbb{C}^{n}$ spanned by the columns of $\mathbf{W} \in \mathbb{C}^{l \times n}$ and $\mathbf{V} \in \mathbb{C}^{m \times n}$, respectively. $\operatorname{dim}\left(\mathcal{M}_{1}\right)=l \leq \operatorname{dim}\left(\mathcal{M}_{2}\right)=m$. Then the principal angles between $\mathcal{M}_{1}$ and $\mathcal{M}_{2}$ are denoted as $0 \leq \theta_{1} \leq \theta_{2} \leq \cdots \leq$ $\theta_{k} \leq \cdots \leq \theta_{l} \leq \frac{\pi}{2}$. The first principal angle $\theta_{1}$ is defined as

$$
\begin{aligned}
\cos \theta_{1} & =\max \left\{\frac{|\langle\mathbf{u}, \mathbf{v}\rangle|}{\|\mathbf{u}\|\|\mathbf{v}\|} \mid \mathbf{u} \in \mathcal{M}_{1}, \mathbf{v} \in \mathcal{M}_{2}\right\} \\
& =\cos \angle\left(\mathbf{u}_{1}, \mathbf{v}_{1}\right),
\end{aligned}
$$

where $\mathbf{u}_{1}$ and $\mathbf{v}_{1}$ are called the principal vectors. The other principal angles and vectors are defined recursively as

$$
\begin{array}{r}
\cos \theta_{k}=\max \left\{\frac{|\langle\mathbf{u}, \mathbf{v}\rangle|}{\|\mathbf{u}\|\|\mathbf{v}\|} \mid \mathbf{u} \in \mathcal{M}_{1}, \mathbf{u} \perp \mathbf{u}_{i}\right. \\
\mathbf{v} \in \mathcal{M}_{2}, \mathbf{v} \perp \mathbf{v}_{i}, \\
i=1, \ldots, k-1\}
\end{array}
$$

where $\mathbf{u}_{i}$ and $\mathbf{v}_{i}$ are the principal vectors of the pair of subspaces.

This is a generalization of the principal (or canonical) angles between two Euclidean subspaces defined in [10] and [11] to Complex subspaces, proposed by the authors of [12]. We propose to measure the distance between precoding matrices $\mathbf{W}$ and $\mathbf{V}$ by the sine of the largest principal angles between the subspace spanned by $\mathbf{W}$ and $\mathbf{V}$, i.e., $d(\mathbf{W}, \mathbf{V})=\sin \left(\max _{k}\left(\theta_{k}\right)\right)$. Intuitively, if the largest principal angle between two subspaces is small, then the two subspaces are nearly linearly dependent. Since $0 \leq \theta_{k} \leq \frac{\pi}{2}, 0 \leq$ $\sin \left(\theta_{k}\right) \leq 1$. For vectors, this corresponds to the sine of the angle between the two lines, i.e., $d(\mathbf{w}, \mathbf{v})=\sqrt{1-\left|\mathbf{w}^{H} \mathbf{v}\right|^{2}}$, as defined in [13].

Ideally, distances between PMIs within a cluster are small and large for PMIs of different clusters. Algorithm 1 describes the procedure of clustering the PMI codebook $\mathcal{C}$ into $m$ clusters.

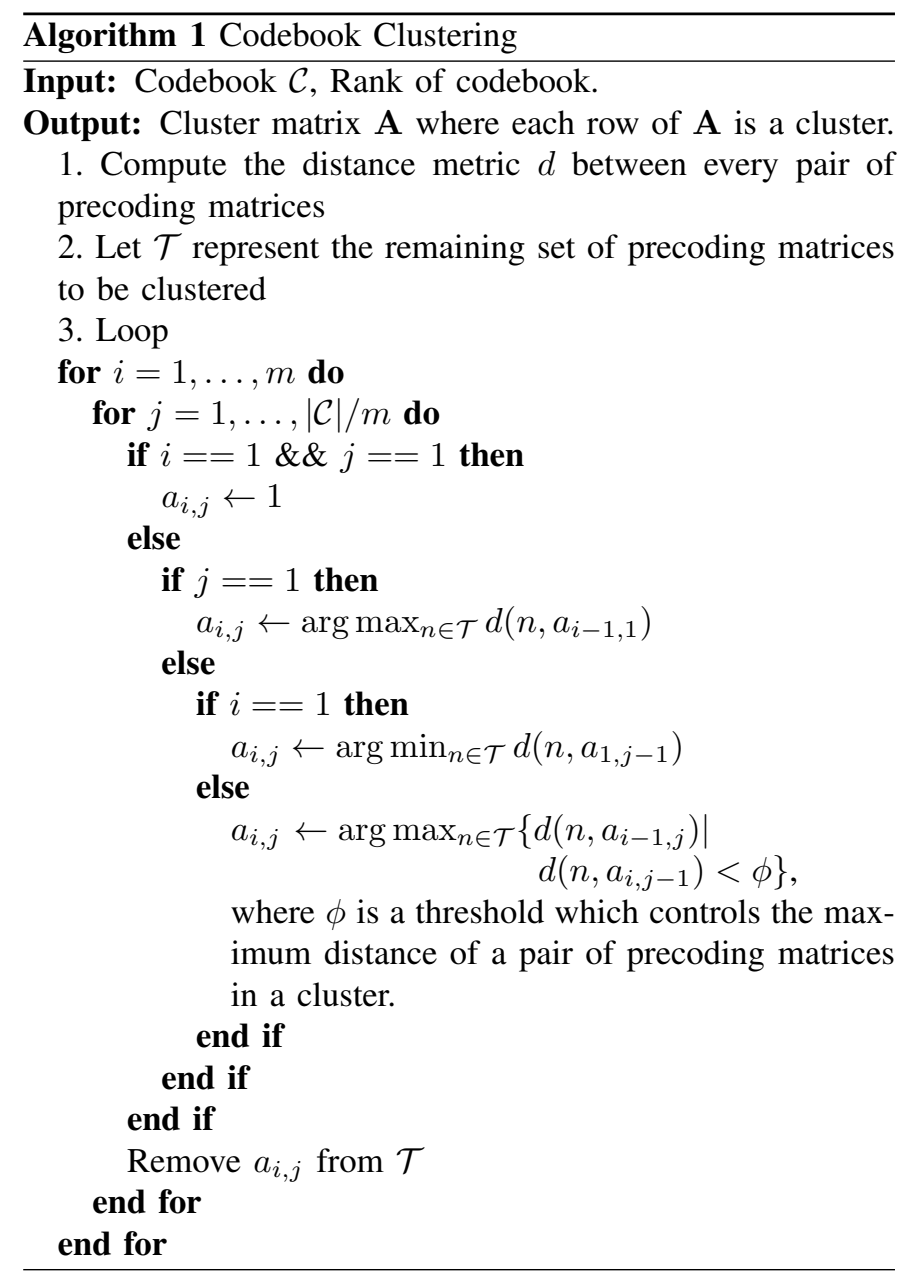

Assume the codebook is to be divided into $m=4$ clusters, an example of a $4 \times 4$ cluster matrix is given by

$$
\mathbf{A}=\left[\begin{array}{cccc}
1 & 10 & 5 & 13 \\
2 & 9 & 8 & 16 \\
3 & 12 & 7 & 14 \\
4 & 11 & 6 & 15
\end{array}\right]
$$

Each element in $\mathbf{A}$ is a precoding matrix index. Each row of $\mathbf{A}$ is a cluster; the distance between the precoding matrices in the same cluster is small. Similarly, the distance between the precoding matrices in the same column of $\mathbf{A}$ is large. Since the codebook based precoding is assumed, codebook clustering can also be done offline.

\section{B. CQI Estimation}

If the rank index of a user $k$ is greater than or equal to 3 , the CQI is given by

$$
\gamma_{k}=\log \operatorname{det}\left(\mathbf{I}+\rho^{2} \mathbf{H}_{k} \mathbf{W}_{k} \mathbf{W}_{k}^{H} \mathbf{H}_{k} / \sigma_{n}^{2}\right),
$$

which is the SU-MIMO rate of that user.

On the other hand, if a user's rank index is less than 3 , it can be co-scheduled with a group of rank-1 or a rank- 2 user. If user $k$ 's preferred PMI is $a_{i, j}$ in matrix $\mathbf{A}$, the group of interfering PMI is estimated by $\left\{a_{1, j}, \ldots, a_{i-1, j}, a_{i+1, j}, \ldots, a_{4, j}\right\}$. We 
call this group the interference PMI cluster. If user $k$ is rank1 , we can estimate the interference from the group of rank-1 PMIs by its interference PMI cluster. We can then estimate the interference from the rank-2 user by finding a rank-2 PMI from the interference PMI cluster such that the total distance between each of the columns of the rank-2 precoding matrix and the user $k$ 's preferred precoding matrix is the largest.

Similarly, if user $k$ is rank-2, we can estimate the interference from the group of rank-1 PMIs by its interference PMI cluster. We can then estimate the interference from the rank-2 user by a precoding matrix from the interference PMI cluster such that the distance is the largest.

Hence, each user can compute the covariance matrix of the estimated interferences plus noise from 1) the rank-1 interference cluster and 2) the rank-2 user to be co-scheduled as

$$
\mathbf{Q}_{k}^{(1)}=\sigma_{n}^{2} \mathbf{I}+\rho_{1}^{2} \mathbf{H}_{k} \widehat{\mathbf{W}} \widehat{\mathbf{W}}^{H} \mathbf{H}_{k}^{H}
$$

and

$$
\mathbf{Q}_{k}^{(2)}=\sigma_{n}^{2} \mathbf{I}+\rho_{2}^{2} \mathbf{H}_{k} \check{\mathbf{W}} \check{\mathbf{W}}{ }^{H} \mathbf{H}_{k}^{H},
$$

respectively, where $\widehat{\mathbf{W}}$ is the row concatenated rank-1 precoding matrices from the interference cluster, $\check{\mathbf{W}}$ denotes the rank-2 precoding matrix and $\rho$ is a scaling factor to satisfy the total transmit power constraint given by (3).

Finally, the CQI for user $k$ with $R_{k}<3$ is given by

$$
\gamma_{k}=\min _{\substack{\mathbf{Q}_{k} \in\left\{\mathbf{Q}_{k}^{(i)}\right\} \\ i=\{1,2\}}} \log \operatorname{det}\left[\mathbf{I}+\rho_{i}^{2} \mathbf{Q}_{k}^{-\frac{1}{2}} \mathbf{H}_{k} \mathbf{W}_{k} \mathbf{W}_{k}^{H} \mathbf{H}_{k}^{H}\left(\mathbf{Q}_{k}^{-\frac{1}{2}}\right)^{H}\right] \text {. }
$$

Intuitively, we are estimating the interference caused to a particular user by either a group of most-likely co-scheduled rank-1 users whose PMIs have the best orthogonality (we named this group the rank-1 interference cluster), or a single rank-2 user, hence covering the possibility of co-scheduling both rank-1 and rank-2 users. This is a more conservative CQI estimation since it covers the worst case scenarios for multirank user scheduling. We can easily extend this if the BS had more transmit antennas: for instance, if the BS has 8 transmit antennas, then the rank- 2 interference can be estimated by the rank-2 interference cluster instead of a single rank-2 user. Since codebook and clustering is pre-designed, the main complexity of CQI estimation comes from computing (8). This interfering cluster estimation works well with our dynamic scheduler proposed in the next section. As we will see in Section V, the sum rate is closely related to the CQI.

\section{DYNAMIC SCHEDULING}

In this section, we propose a user selection algorithm with the capability to dynamically switch between SU-MIMO and MU-MIMO. Moreover, it can co-schedule rank-1 and rank-2 users when operating on MU-MIMO mode.

The algorithm is described as follows. Based on the reported rank index from all users, the scheduler will divide the users into two groups. The SU-MIMO group contains all the users with rank index greater than or equal to 3 and the MU-MIMO group contains all the users with rank index less than 3 . Then, the scheduler will select a user with the highest reported rate (or CQI) from the SU-MIMO group and select a subset of users such that the estimated sum rate is the largest. The system will operate in MU-MIMO mode, if the highest sum rate is larger than the highest rate reported by SU-MIMO users; and operate in SU-MIMO mode otherwise.

The scheduler will determine co-scheduled users from the MU-MIMO group as follows. First select the user with the highest CQI; then select the next user such that the total distance between its preferred PMI and each of the previous user's preferred PMI is the largest. Once a new user is added to the set, remove all other users from the remaining set whose preferred PMI is the newly added user's preferred PMI. Repeat until the total rank is $M$ or no more users are available.

Our proposed scheduler first tries to select the next user with the same rank as the previous user and switches to a different rank if it cannot find a suitable user of the same rank. The switching between rank-1 and rank-2 is as follows; if the previous user picked is of rank- 2 and the scheduler wishes to switch to a rank-1 user, it should pick the user such that the total distance between its preferred PMI and each of the columns of previous user's preferred PMI is the largest. Similarly, if the previous user picked is of rank-1 and the scheduler wishes to switch to a rank-2 user, then the BS should pick the next user such that the total distance between each of the previous user's PMI and each of the columns of its preferred PMI is the largest.

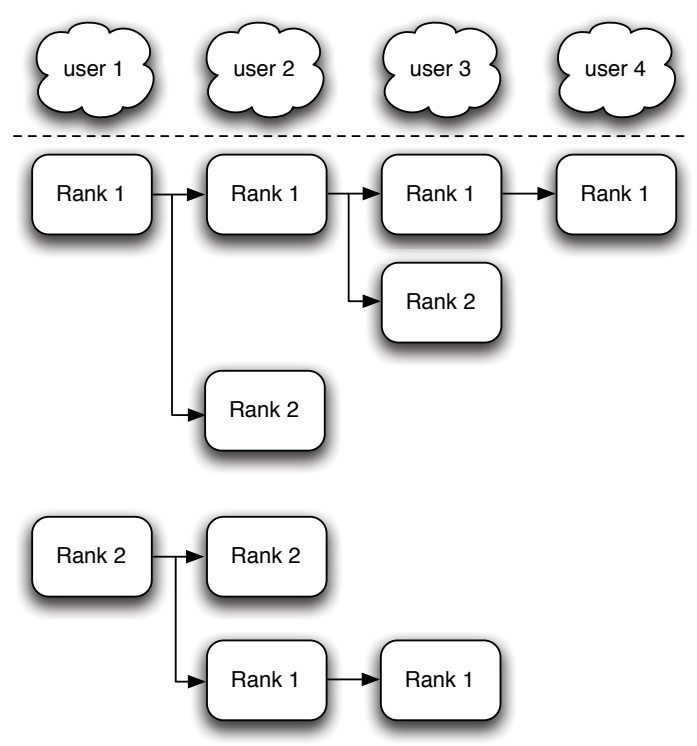

Fig. 2. MU-MIMO Scheduling Scenarios

Figure 2 summarizes the MU-MIMO scheduling scenarios of our scheduler. It differs from the other proposed schedulers in the literature. First, we have proposed a CQI estimation method based on interference cluster. If every user is of rank-1, this can be thought of as a extension of [6] where 
the difference is that we do not restrict to only 2 users. And compared with [3], our proposed scheduler finds the best orthogonality between the preferred PMIs instead of the channel itself. But more importantly, our model do not restrict to only rank-1 users and we have proposed a scheduling framework to switch between SU-MIMO and MU-MIMO and to schedule multi-rank Multi-User transmissions, without additional multi-user feedback information.

Let $\mathcal{S}$ represent the set of candidate users of the same rank as the current user and $R_{\text {total }}$ represent the total rank of the selected user set. Algorithm 2 describes our proposed scheduler.

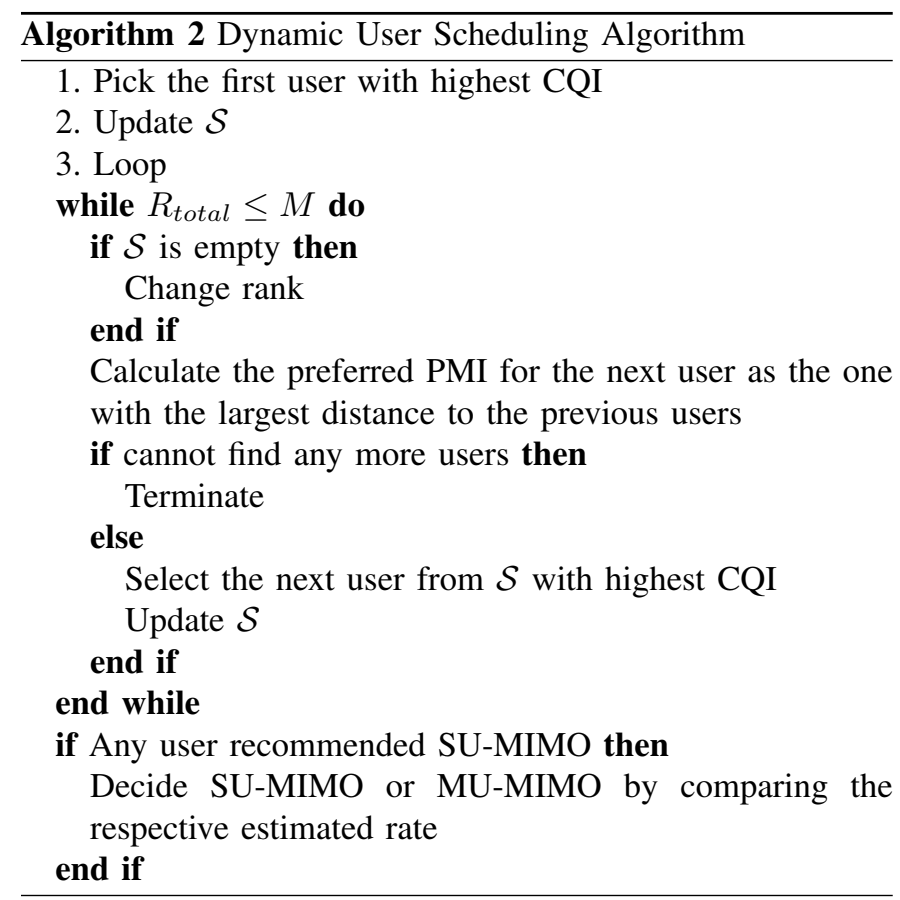

Our proposed scheduler has low complexity since codebooks are pre-designed. We can compute the distances between each precoding matrix and store them in a lookup table at the base station. This significantly reduces the scheduling complexity as the only complexity of the scheduler comes from sorting the users according to their CQI and searching for the next user.

\section{Achievable Sum Rate}

The interference plus noise at user $k$ can be written as

$$
\sum_{\substack{j \in \mathcal{U} \\ j \neq k}} \rho \mathbf{H}_{k} \mathbf{W}_{j} \mathbf{x}_{j}+\mathbf{n}_{k}
$$

We assume each user use ML receiver. In this case, the sum achievable rate is given by

$$
C=\mathbf{E}\left[\sum_{k \in \mathcal{U}} \log \operatorname{det}\left(\mathbf{I}+\mathbf{Q}_{k}^{-\frac{1}{2}} \widetilde{\mathbf{H}}_{k} \widetilde{\mathbf{H}}_{k}^{H}\left(\mathbf{Q}_{k}^{-\frac{1}{2}}\right)^{H}\right)\right],
$$

$\widetilde{\mathbf{H}}_{k}=\mathbf{H}_{k}\left(\rho \mathbf{W}_{k}\right)$ is the effective channel of user $k$ and

$$
\mathbf{Q}_{k}=\sigma_{n}^{2} \mathbf{I}+\mathbf{H}_{k} \sum_{\substack{j \in \mathcal{U} \\ j \neq k}} \rho^{2} \mathbf{W}_{j} \mathbf{W}_{j}^{H} \mathbf{H}_{k}^{H}
$$

is the covariance matrix of the interference plus noise at user $k$. The above expressions represent the sum rate of the selected set of users.

Due to the high complexity of ML detection, we also consider the simpler Linear MMSE receiver. In this case, the sum rate is given by

$$
C=\mathbf{E}\left[\sum_{k \in \mathcal{U}} \sum_{i=1}^{R_{k}} \log \left(1+\check{\mathbf{h}}_{k_{i}}^{H} \mathbf{K}_{k_{i}}^{-1} \check{\mathbf{h}}_{k_{i}}\right)\right],
$$

where $\check{\mathbf{h}}_{k_{i}}$ is the $i$-th column of the matrix $\check{\mathbf{H}}_{k}=\mathbf{Q}_{k}^{-\frac{1}{2}} \widetilde{\mathbf{H}}_{k}$ and

$$
\mathbf{K}_{k_{i}}=\mathbf{I}+\sum_{j \neq i} \check{\mathbf{h}}_{k_{j}} \check{\mathbf{h}}_{k_{j}}^{H} .
$$

\section{NUMERICAL RESULTS}

In this section, we present our simulation results. We assume that the number of transmitting antennas at the base station is fixed at 4 and the number of receiving antennas for user $k$ is $N_{k} \in\{1,2,3,4\}$ with equal probability. Moreover, we assume that the total transmission power at the BS is constant and equal power allocation between the users for simplicity.

We will compare the performance of our proposed scheduler with the best-companion user pairing method proposed in [9], which is briefly discribed below. Each user will feedback the best-companion PMI in addition to its preferred PMI by computing

$$
\beta(k)=\arg \min _{\mathbf{C} \in \mathcal{C}^{\left(R_{k}\right)}} \operatorname{det}\left(\mathbf{I}+\mathbf{H}_{k} \mathbf{C C}^{H} \mathbf{H}_{k}^{H}\right),
$$

where $\mathcal{C}^{\left(R_{k}\right)}$ is the codebook of rank $R_{k}$.

Since there are only two users, the covariance matrix of the interference plus noise at user $k$ is given by

$$
\mathbf{Q}_{k}=\sigma_{n}^{2} \mathbf{I}+\rho^{2} \mathbf{H}_{k} \mathbf{W}_{\beta(k)} \mathbf{W}_{\beta(k)}^{H} \mathbf{H}_{k}^{H},
$$

where $\mathbf{W}_{\beta(k)}$ is the best-companion precoding matrix for user $k$. Therefore, the CQI for user $k$ is given by

$$
\gamma_{k}^{B C}=\log \operatorname{det}\left[\mathbf{I}+\rho^{2} \mathbf{Q}_{k}^{-\frac{1}{2}} \mathbf{H}_{k} \mathbf{W}_{k} \mathbf{W}_{k}^{H} \mathbf{H}_{k}^{H}\left(\mathbf{Q}_{k}^{-\frac{1}{2}}\right)^{H}\right] .
$$

At the BS, the scheduler will find a pair of users (of the same rank) such that the preferred PMI and the best-companion PMI is $\alpha, \beta$, respectively for the first user and $\beta, \alpha$ for the second user. In other words, there is an exact match such that the two users are mutually optimal for each other. If the BS cannot find such a pair, the BS will schedule to SU-MIMO.

Figure 3 shows the sum rate vs. SNR. The number of users is fixed at 100. As we can see from this figure, the sum rate increases with SNR. Our proposed scheduler outperforms bestcompanion user pairing for both the ML and LMMSE receivers, where the best-companion user pairing uses 4 bits for 


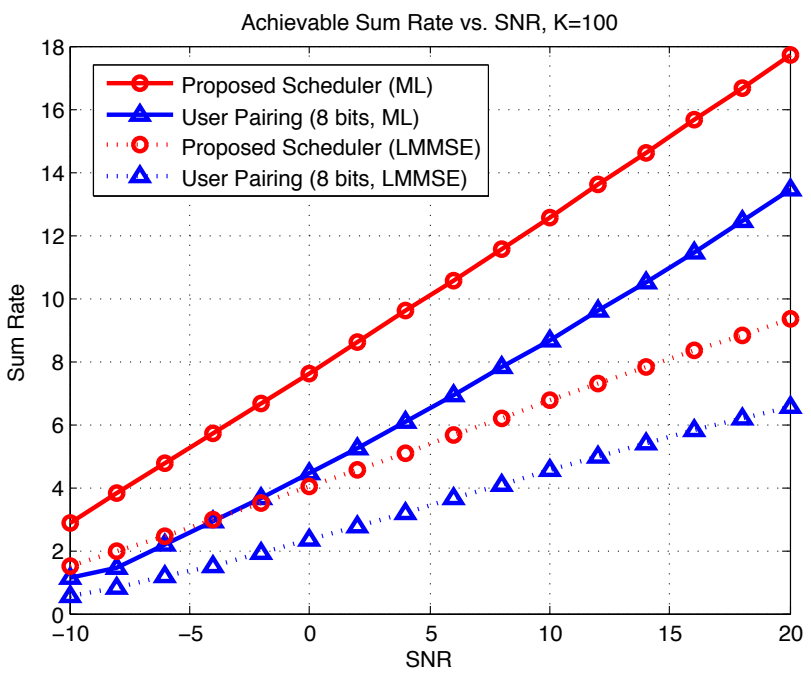

Fig. 3. Sum Rate vs. SNR, $M=4$, Number of users is fixed at 100

feeding back the preferred PMI and the best-companion PMI, respectively. As we mentioned before, the best-companion user pairing scheme has no scheduling flexibility across different ranks and only limits to a user pair. This result is remarkable when we consider that our proposed scheduler requires less feedback compared to best-companion user pairing.

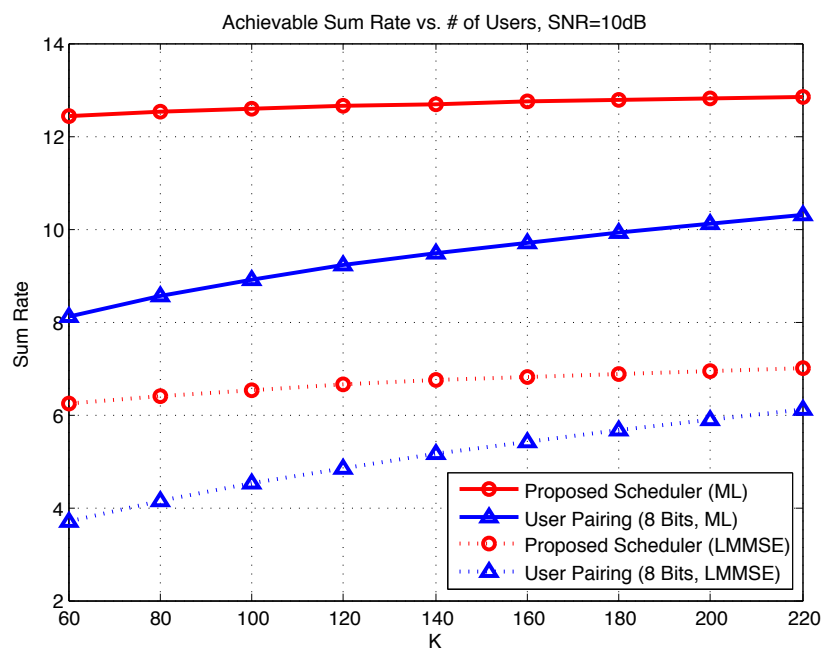

Fig. 4. Sum Rate vs. \# of users, $M=4$, SNR is fixed at $10 \mathrm{~dB}$

Figure 4 shows the sum rate vs. the number of users for the case of SNR $=10 \mathrm{~dB}$. Our proposed scheduler outperforms to the best-companion user pairing method for both the ML and LMMSE receivers. It is interesting to note that the sum rate does not increase much as the number of users increase at $\mathrm{SNR}=10 \mathrm{~dB}$. This is due to the fact that the number of feedback bits is fixed for each user. When the codebook is fixed and each user feeds back an index to represent their channel from this codebook, multi-user diversity is reduced.

\section{CONCLUSION}

In this paper, a general MU-MIMO system model with limited feedback is considered where each user has different number of receiving antennas and operates on different ranks. A CQI estimation method are proposed for scheduling purposes based on the clustering algorithm for the 4-bit 4-Tx LTE codebook. Moreover, a dynamic scheduling algorithm to support MU-MIMO is proposed based on the user RI/PMI/CQI and numerical results show that the performance is better compared with the best-companion user pairing scheme. In summary, we considered a general MU-MIMO model that many people in the past have overlooked and showed that if designed properly, good performance can be achieved without the feedback of multi-user information to support dynamic MU-MIMO scheduling. Instead of using additional bits to feedback multi-user information, we can increase the feedback granularity in the spatial domain to better capture the users' CSI.

\section{ACKNOWLEDGMENT}

This work is supported by the Australian Research Council Linkage Project in collaboration with NEC Australia. The authors would like to thank Chaitanya Rao, Phong Nguyen, Gordon Gay and Dobrica Vasic from NEC Australia for their helpful discussions.

\section{REFERENCES}

[1] M. Sharif, B. Hassibi, "On the capacity of MIMO broadcast channels with partial side information," IEEE Trans. on Information Theory, vol. 51, no. 2, pp. 506-522 Feb. 2005

[2] 3GPP, "Evolved Universal Terrestrial Radio Access (E-UTRA); Physical channels and modulation", 3GPP TS 36.211 version 10.0.0 Release 10.

[3] T. Yoo and A. Goldsmith, "On the optimality of multiantenna broadcast scheduling using zero-forcing beamforming," IEEE Trans. on Selected areas in Communications, vol. 24, no. 3, pp. 528-541, March 2006

[4] T. Yoo, N. Jindal and A. Goldsmith, "Multi-antenna downlink channels with limited feedback and user selection," IEEE Trans. on Selected Areas in Communications, vol. 25, No. 7, Sept. 2007

[5] M. Trivellato et al., "User selection schemes for MIMO broadcast channels with limited feedback", IEEE Vehicular Tech. Conf., 2007

[6] Shaofeng Du et al., "Adaptation between codebook based SU-MIMO and MU-MIMO with single MCS feedback in LTE," 3rd International Conference on Broadband Network \& Multimedia Technology, ICBNMT 2010

[7] Xin Xia et al., "Joint user pairing and precoding in MU-MIMO broadcast channel with limited feedback," IEEE Communications Letters, vol. 14, no. 11, pp. 1032-1034, Nov. 2010

[8] Yinggang Du et al., "Evaluation of PMI feedback schemes for MUMIMO pairing", IEEE Systems Journal, vol. 4, no. 4, pp. 505-510, Dec. 2010

[9] "Best companion reporting for improved single-cell MU-MIMO pairing”, Alcatel-Lucent, R1-090926, R1-091307, R1-092031, R1-092546, R1-093333

[10] Sheng Jiang, "Angles between Euclidean subspaces", Geometriae Dedicata, vol. 63, no. 2, pp. 113-121, Nov. 1996

[11] Jianming Miao and Adi Ben-Israel, "On principal angles between subspaces in $\mathbb{R}^{n}$ ", Linear Algebra and its Applications, vol. 171, pp. $81-98$, July 1992

[12] A. Galántai and Cs. J. Hegedüs, "Jordan's principal angles in complex vector spaces", Numerical Linear Algebra with Applications, vol. 13, no. 7, pp. 589-598, 2006

[13] D. J. Love, R. W. Heath and T. Strohmer, "Grassmannian beamforming for multiple-input multiple-output wireless systems," IEEE Trans. on Information Theory, vol. 49, no. 10, pp. 2735-2747, Oct. 2003 